\title{
Uso de resíduos de madeira como alternativa de melhorar as condições ambientais em sistema de reflorestamento
}

\author{
Kátia Fernanda Garcez MONTEIRO¹, Dirse Clara KERN², Maria de Lourdes Pinheiro RUIVO³, Tarcísio \\ Ewerton RODRIGUES ${ }^{4}$, José Luis Said COMETTI5
}

\section{RESUMO}

A baixa tecnologia empregada no manejo do solo e utilização do fogo para o plantio de espécies ou cultivares florestais na Amazônia, tem sido apontada como a causa principal das áreas alteradas em sistemas florestais, resultando em erosão, poluição hídrica perda de nutrientes e da biodiversidade. Assim, pode-se hipotetizar que uma área alterada, seja em que ambiente ou tipo de exploração a que esteja submetida, estaria em fase de recuperação quando o teor de matéria orgânica no solo estiver aumentando. Tal condição pode ser medida, através de indicadores biológicos do solo: matéria orgânica e teores de nutrientes, e atividade da biomassa microbiana, e também influencia a qualidade e desenvolvimento (diâmetro e altura das árvores ou biomassa aérea) dos espécimes plantados. As áreas atualmente em recuperação ou recuperadas em suas propriedades edafológicas podem ser comparadas, em termos de bioindicadores, a solos enriquecidos com material orgânico proveniente de manejo pretérito, como em áreas de reflorestamento instaladas em áreas de "Terra Preta". Essa comparaçáo, além de validar os indicadores de qualidade do solo, auxiliará estudos que contemplem a utilização racional, seja de florestas naturais ou florestas plantadas.

PALAVRAS-CHAVE: solos amazônicos, indicadores ambientais, resíduos de madeira, floresta plantada de paricá.

\section{Use of waste wood as an alternative to improve environmental conditions in the system of reforestation in Tailândia-PA}

\begin{abstract}
A substantial portion of soils in the Amazon has low fertility and high acidity indexes. The low levels of technology used in land management and in the use of fire has been pointed out as the main cause of alteration of areas covered by forests. Inadequate soil management, either while planting forest species or agricultural crops, leads to environmental modifications, such as erosion, water pollution and loss of nutrients and biodiversity. Despite the difficulty to measure the degree of recovery of those areas, we may assume that an altered area, whatever environment or land use type it is submitted, would be in recovery phase when the status of its organic matter is improving. This can be measured through soil biological indicators such as levels of organic matter and nutrients, and activity of the microbiological biomass. It also influences the quality of the planted specimens in terms of tree diameter and height, and aerial biomass. Areas currently under recovery or recovered in their edaphological properties can be compared, through the use of bioindicators, to soils enriched with organic material from organic soil management in the past, such as forest systems planted in areas of black earths. This comparison, besides validating soil quality indicators, will be helpful in studies involving rational use of both natural and planted forests.
\end{abstract}

KEYWORDS: Amazon soils, environmental indicators, wood residues, paricá plantations

\footnotetext{
1 Universidade Federal Rural da Amazônia, E-mail: kfgarcez@hotmail.com

2 Museu Paraense Emílio Goeldi, E-mail: kern@museu-goeldi.br

3 Museu Paraense Emílio Goeldi, E-mail: ruivo@museu-goeldi.br

${ }^{4}$ Embrapa Amazônia Oriental, E-mail: tarcisio@cpatu.embrapa.br

${ }^{5}$ Secretaria de Meio Ambiente de Pernambuco, E-mail: jlscometti@yahoo.com
} 


\section{INTRODUÇÃO}

Nos últimos anos vem crescendo o número de empresários rurais que, além da adoção de cultivos agrícolas, optam em fazer projetos de reflorestamento com vistas à reposição florestal, adotando cultivos simples ou consorciados. Nesse sentido, atendem à legislação ambiental e contribuem para o melhoramento das propriedades físicas e químicas dos solos, principalmente em zonas tropicais.

A utilização de sistemas agroflorestais implica na escolha de espécies ecológica e economicamente apropriadas às finalidades desejadas. Entretanto, na Amazônia, ainda são poucas as informaçóes acerca do comportamento de espécies florestais utilizadas nesses sistemas, como é o caso do paricá (Schizolobium amazonicum). Um exemplo desta problemática pode ser verificado na mesorregião do nordeste paraense, onde até o ano de 2002 foram implantados cerca de 35 projetos de reposição florestal. Os SAFs vêm apresentando uma crescente demanda, com área ocupada de 34.864 ha (Falesi \& Galeão 2002), principalmente em áreas alteradas. A escolha da espécie paricá para a implantação de sistemas agroflorestais atinge um percentual de $38 \%$ na preferência de empresas madeireiras. Entre os aspectos atrativos estấo: por pertencer à flora amazônica, ocorrendo nos maciços florestais paraenses, pelo rápido crescimento, pela facilidade de obtenção de sementes e baixo custo de implantação, sendo bastante utilizada para a fabricaçáo de laminados e compensados para a indústria moveleira.

Por outro lado, o avanço tecnológico tem catalisado uma gama de novas técnicas e vem demonstrando que os diversos setores da agricultura não podem mais serem vistos de forma homogênea, no que tange à mensuração das variáveis pesquisadas. Tais técnicas aumentaram substancialmente a quantidade e a qualidade de informação ambiental que pode ser empregada para reconstruir novos espaços produtivos. Isto tem colocado a variação temporal e espacial como elementos chaves para a melhor aplicação e conhecimento da produção agrícola, principalmente em sistemas agroflorestais em zonas tropicais (Vieira et al. 2000). Assim, o desenvolvimento de pesquisas voltadas ao melhoramento da fertilidade dos solos é imprescindível para viabilizar uma agricultura sustentável nos trópicos. Sob esta ótica, o aumento da produção nem sempre será o enfoque único, devendo estar associado à otimização das condiçôes ambientais e a preservação do potencial de uso diversificado dos solos e dos sistemas de plantio florestal.

Vale ressaltar que o desenvolvimento de processos que permitam transformar resíduos e subprodutos da atividade madeireira em compostos com reatividade e estabilidade apropriadas para o uso como adubos orgânicos, é altamente desejável e estratégico para o setor florestal brasileiro. Sobretudo, estará atendendo a legislação florestal e ao mesmo tempo proporcionando condiçôes para que produtos agrícolas cultivados mediante estas práticas consideradas sustentáveis sejam certificados. As leis atuais sobre desmatamento na Amazônia permitem que $20 \%$ da área da propriedade sejam desmatadas, sendo exigida a obtençáo de autorizaçóes de desflorestamento - ADMs. A regiâo amazônica representa uma parte muito importante na área ambiental a nível mundial, devido a sua grande reserva natural tropical, e porque atualmente desmata-se a floresta para fins comerciais, contribuindo para a destruição dos recursos naturais (Kato, 2003). Estudos sobre uso de sistemas agroflorestais na conservação ao meio ambiente estão sendo realizados em diversas localidades da regiâo (Cordeiro, 1999; Monteiro, 2004; Ribeiro, 2006), demonstrando os grandes benefícios sociais e ambientais com o reflorestamento de espécies nativas e manejo de floresta secundária para manter a produtividade sem alterar o ecossistema.

Assim, o presente trabalho visa avaliar o desenvolvimento da espécie Schizolobium amazonicum parahyba var. (paricá) em dois diferentes sistemas de manejo do solo com e sem adição de resíduos de madeira e a sua correlaçáo com a qualidade do solo nas áreas de estudo.

\section{MATERIAL E MÉTODOS}

Localizado na mesorregião do Nordeste Paraense, a cerca de $220 \mathrm{~km}$ de Belém (Figura 1), o Município de Tailândia tem sua posiçáo definida pelas coordenadas geográficas $2^{\circ} 36^{\prime}$ e $3^{\circ}$

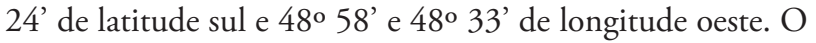
Município possui superfície de $4.430 \mathrm{Km}^{2}$ e uma população estimada em 49.412 habitantes (IBGE, 2004).

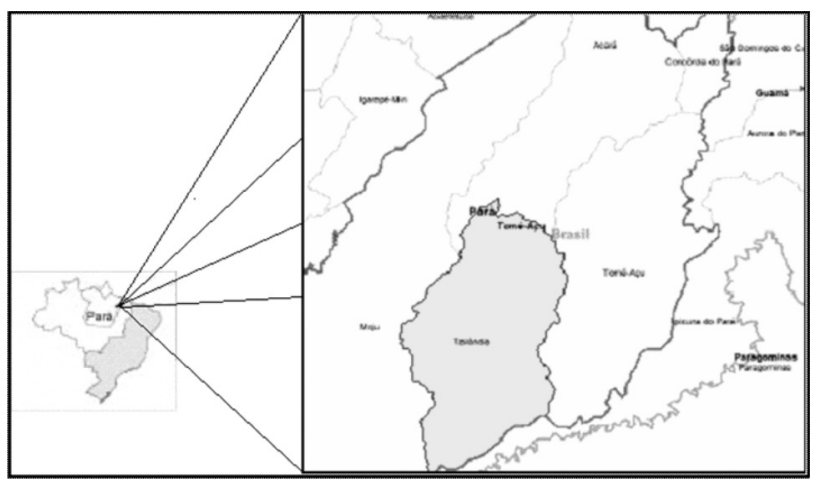

Figura 1 -Localização do Município de Tailândia - Pará. Fonte: IBGE (2000).

Em Tailândia, cidade de muita exploração e beneficiamento de madeira, torna-se muitas vezes problemática a estocagem e reutilizaçấo dos resíduos dessa indústria. Ao lado de Paragominas, Tailândia é o município que detêm uma das mais altas taxas de ocorrência de serrarias no estado do Pará. Por conseguinte, a pressão sobre suas áreas florestais é grande. Recentemente, essa pressáo aliada à necessidade de identificar alternativas para a continuidade da exploração 
florestal na regiáo, tem resultado no surgimento de projetos alternativos para produçáo de madeira por parte de algumas empresas. A Empresa Tailâminas, por exemplo, desde o ano 2000 tem realizado trabalho experimental utilizando resíduos da indústria madeireira na implantação de um sistema diferenciado em áreas de reflorestamento com o plantio de pupunheira (Bactris gasipaes) associada ao paricá. A primeira espécie é destinada à fabricação de palmito, e a segunda para a fabricaçáo de celulose/ou carvão. Para este plantio foram usados resíduos de madeira na preparaçáo do solo, como fontes de matéria orgânica e nutrientes. De uma área total plantada de 18 ha de paricá foram selecionadas duas parcelas de 1 ha, sendo que na primeira, adicionou-se aproximadamente $6 \mathrm{~m}^{3}$ de resíduo de madeira, e na segunda, manteve-se o solo sem qualquer tipo de adubação (Figura 2). Adotou-se o plantio direto com aproximadamente 1.400 mudas de paricá. Para a identificação das características dendométricas do paricá foram amostradas 629 árvores na área com e sem a aplicação de resíduos de madeira, totalizando 1.258 amostragens.

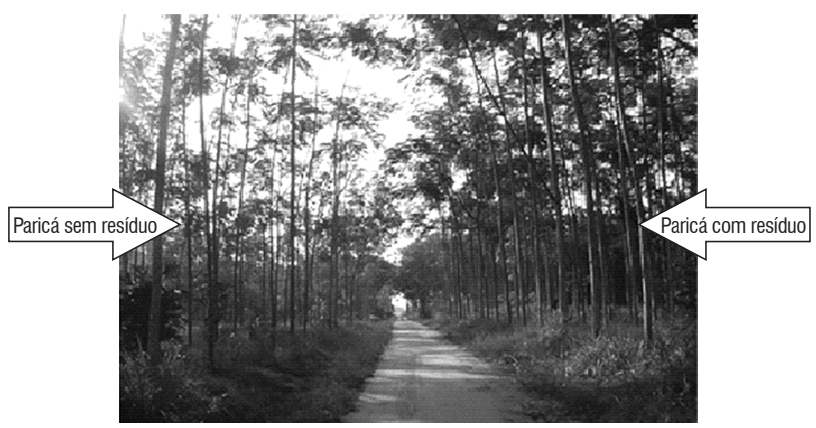

Figura 2 -: Área experimental da empresa Tailâminas, com e sem utilização de resíduos de madeira. Foto:Kern (2004).

Foi utilizado como medida dendométrica o DAP (diâmetro a altura do peito), para avaliar o grau de desenvolvimento do plantio como indicador da eficiência do uso de resíduos de madeira. O DAP foi obtido através da circunferência da árvore, medida com uma fita métrica padrão, no mês de agosto de 2003, quando as plantas apresentavam idade de 52 meses.

\section{RESULTADOS E DISCUSSÃO}

Os dados de desenvolvimento do paricá apresentaram distribuição normal, conforme as Figuras 3 e 4 . Para a área que recebeu adição de resíduos, apenas 42 indivíduos $(6,7 \%)$ não sobreviveram. Por outro lado, na área que não recebeu material orgânico, o percentual de mortalidade aumentou para $20 \%$. As árvores que receberam cobertura no solo mostraram os maiores valores em DAP, com 78,6\% delas exibindo valores entre 15 e $27 \mathrm{~cm}$ (Figura 3). Nesse mesmo intervalo de DAP, porém na área sem cobertura, o percentual foi de 51,7\% (Figura 4), evidenciando grande variabilidade na área de estudo. Esse estudo mostrou, ainda, que na área com adiçáo de resíduos, alguns indivíduos conseguiram atingir $30 \mathrm{~cm}$ de DAP em 4 anos de crescimento (dados náo mostrados). Segundo Loureiro (2000), o paricá pode atingir até $50 \mathrm{~cm}$ de DAP em 8 anos. O considerável crescimento das plantas de paricá pode ser explicado pelo importante papel que a camada de resíduos de madeira desempenhou no solo, favorecendo o incremento da matéria orgânica e conseqüentemente melhor liberação de nutrientes essenciais para a cultura de paricá.

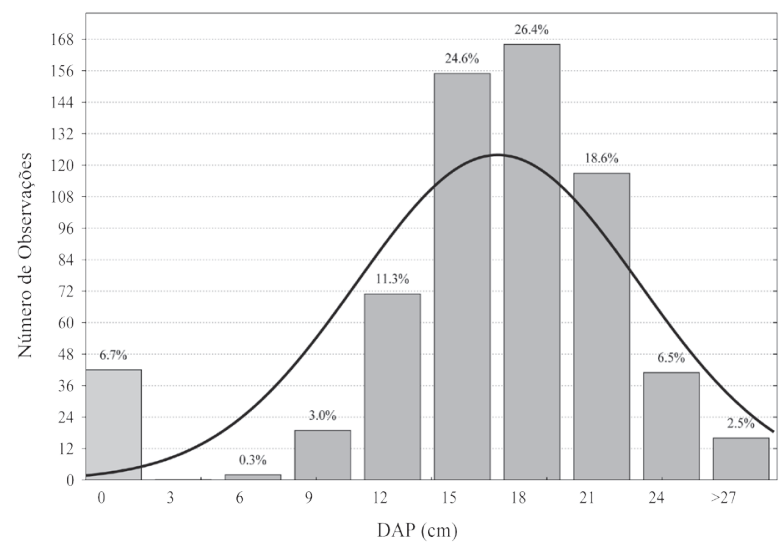

Figura 3 -Desenvolvimento do paricá com adição de resíduo de madeira. Fonte: (Monteiro, 2009)

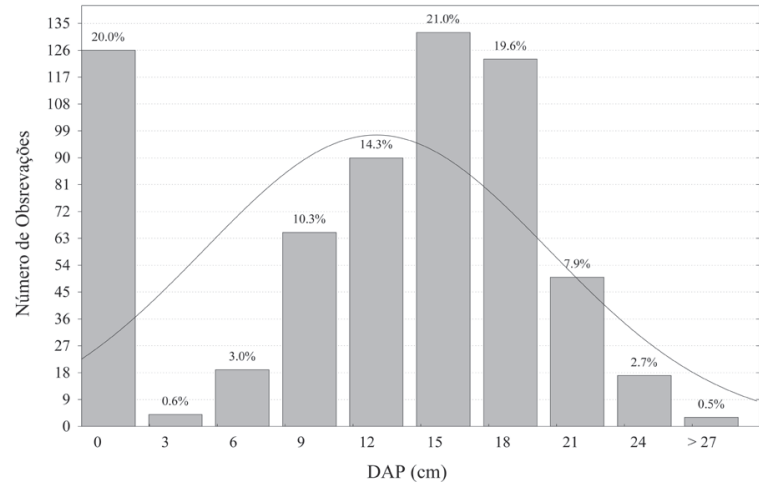

Figura 4 - Desenvolvimento do paricá sem resíduo de madeira. Fonte: (Monteiro,2009)

Os resultados apresentados corroboram com (Marques, 1990; Silva, 1997; Cordeiro, 1999). Esses autores, estudando a desempenho diferencial de crescimento do paricá sob diferentes regimes de preparaçáo de área, no estado do Pará, verificaram que o paricá se adapta perfeitamente ao sistema de manejo de solo utilizando resíduos orgânicos como cobertura. Ruivo et al. (2006) avaliaram a influência positiva no preparo do solo com adição de material orgânico sobre o 
desenvolvimento do paricá, mesmo em associação com outras espécies vegetais.

Em relação à composição química dos solos com adição de resíduos de madeira, os perfis de solo com e sem adiçấo de resíduos de madeira demonstraram variaçóes entre si. Os valores de cálcio para os perfis sem adição de resíduos de madeira foram de valores semelhantes a $1,5 \mathrm{cmolc}^{-\mathrm{dm}^{-3}}$ nos horizontes superficiais, decrescendo em profundidade.

A distribuição dos valores de cálcio nos solos com adição de resíduos de madeira, alcançaram melhores resultados no horizonte A com 2,6 cmolc. $\mathrm{dm}^{3}$. Estes valores mais elevados representam a contribuiçáo do material adicionado ao solo em elevar este nutriente (Tabela 1).

Para os valores de $\mathrm{pH}$ os perfis com adição de resíduos de madeira, foram significativamente superiores. Cujos resultados indicam a eficiência da matéria orgânica adicionada ao solo, proporcionando diminuição considerável de sua acidez nas profundidades estudadas.

A disponibilidade de maiores concentraçóes de cálcio nestes solos a partir do material vegetal utilizado como cobertura, influenciou sobremaneira nos valores de $\mathrm{pH}$, o que pode ser explicado também, pela presença de cargas dependentes de $\mathrm{pH}$ e, estas associadas diretamente à matéria orgânica, originadas da associaçáo e dissociaçáo do íon de $\mathrm{H}+$ com radicais dos grupos carboxílicos e fenólicos (Primavesi,1988; Raij, 1991).

Os resultados encontrados para a soma de bases foram médios, visualizados na tabela 1 . as profundidades estudadas, apresentaram maior eficiência para os perfis com adiçáo de resíduos de madeira (Monteiro et al.,2006).

Abreu Jr et al. (2001) apresentaram que as classes destes solos apresentam razoáveis capacidades de troca de cátions, representando, portanto, a graduação da liberação de vários nutrientes, favorecendo de certa maneira a manutenção da fertilidade por um prolongado período de tempo e reduzindo ou evitando a ocorrência tóxica de outros elementos no solo.

Um exemplar do comportamento dos solos com adição de material orgânico são os solos de Terra Preta Arqueológica (TPA), que seriam antigos assentamentos indígenas formados a partir da ocupação humana pretérita. Para Kern (1996), os grupos pré-históricos formadores de TPA tinham sua subsistência baseada no cultivo de grãos e raízes, complementado com caça, pesca e coleta, permitindo uma permanência mais prolongada no local. Os resíduos orgânicos decorrentes dessa prática cultural são os responsáveis diretos pela concentração de elementos químicos nas TPA, como $\mathrm{Zn}, \mathrm{Mn}, \mathrm{Cu}, \mathrm{Ca}, \mathrm{Mg}$ e P. Folhas de palmeiras, frutos de açaí,

Tabela 1 - Características químicas de solos com e sem resíduos de madeira

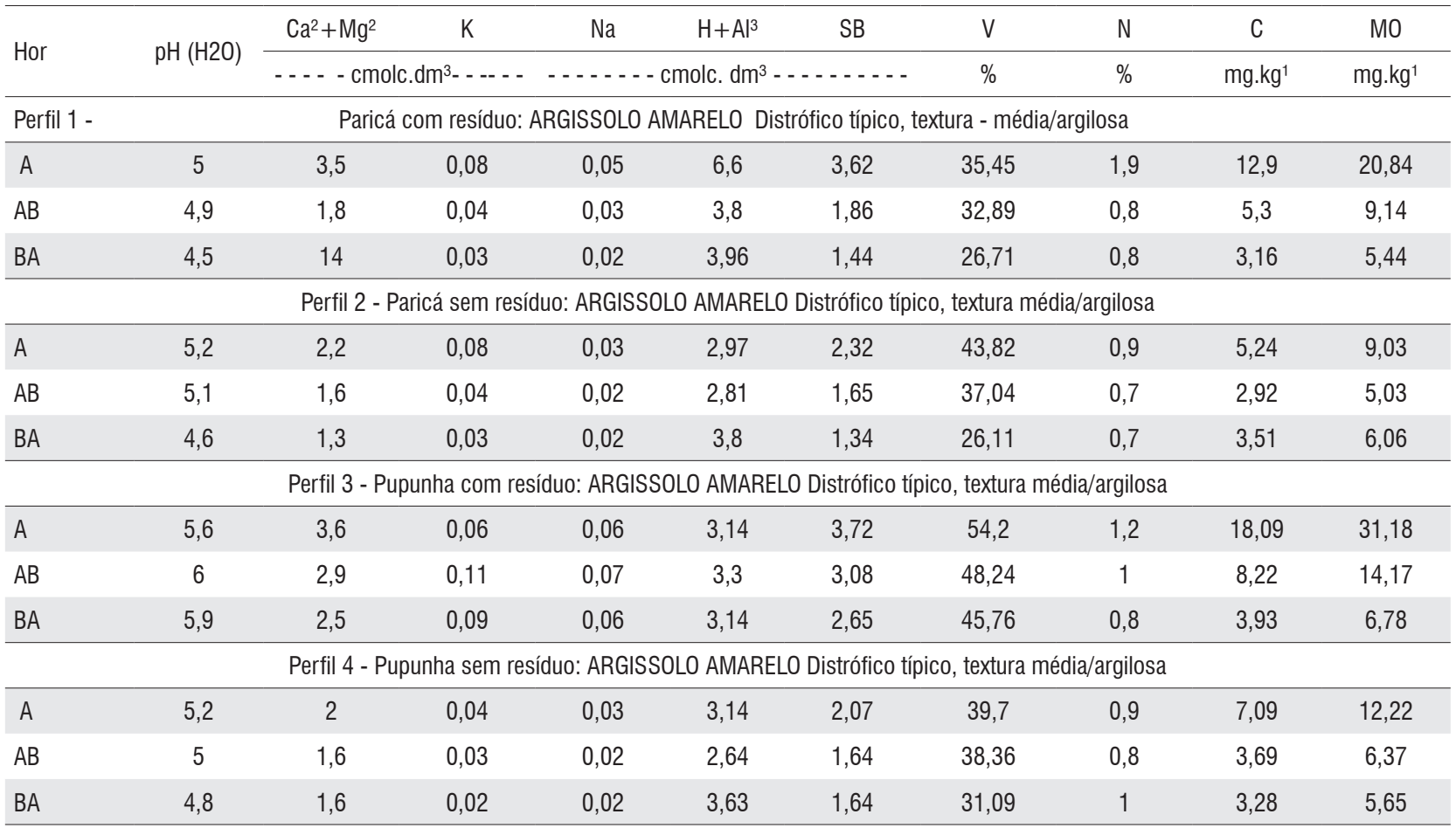

Fonte : Monteiro, 2004 
cascas de mandioca, ossos, conchas, carapaças de quelônios e outros, produzem grande volume de material que é descartado.

Kern et al., (2006) na regiâo de Caxiuanã-PA, os elementos $\mathrm{Zn}$ e Mn em TPA ocorrem em teores superiores a 50 e 700 mg.kg-1 de solo, respectivamente, muito elevados quando comparados com o Latossolo Amarelo da Amazônia, que apresenta teores em torno de 2 a $3 \mathrm{mg} \cdot \mathrm{kg}^{1}$. A hipótese de que os elevados teores desses elementos em TPA sejam oriundos de resíduos de origem vegetal e náo de origem animal, pode ser reforçada, também, pelos elevados teores destes elementos nos resíduos de lâmina de madeira, observados no presente estudo.

\section{CONCLUSÕES}

Os ganhos relativos com adição de resíduos de madeira ao solo, sob um sistema de reflorestamento com a cultura de paricá, ficaram evidentes com maior desempenho da cultura sob esta prática agrícola, demonstrado através dos índices de maior sobrevivência e DAP. A utilização de SAFs que incluem a utilização de espécies madeireiras, como alternativa para a recuperação do ambiente florestal no Estado do Pará, constitui-se em estratégia para minimizar a degradação ambiental, crescente pelo uso desordenado destes recursos.

Os resultados referentes à prática de utilização de resíduos orgânicos como cobertura no solo, pode indicar uma nova forma de manejo dos solos Amazônicos e ao mesmo tempo contribuir para a melhoria da qualidade de vida da população da regiāo.

\section{AGRADECIMENTOS}

Á Secretaria de Meio Ambiente do Estado do Pará. À Superintendência do desenvolvimento da Amazônia. Aos parceiros fundamentais: Prefeitura Municipal de Tailândia - Secretaria de Agricultura e a Empresa Tailâminas Plac. Universidade Federal Rural da Amazônia, Embrapa, Museu Paraense Emílio Goeldi.

\section{BIBLIOGRAFIA CITADA}

Araújo, E. A.; Lani, J. L.; Amaral ,E. F.; Guerra, A. 2004. Land use and physical and chemical properties of a distrophic Yellow argissol in the western amazon region. Rev. Bras. Ciênc. Solo. 28 (2): 307-315 (In portuguese whit abstract in english).

Basso. L.F.C.; Kimura.,H. 2008. The black earth eco-system recreating what used to exist: the black earth of the amazon indians. Rev. Ciênc. Admin. 14 (2): 230-250 ( In portuguese whit abstract in english).

Cordeiro, I.M.C. 1999. Differential Growth Performance of Species Schizolobium amazonicum Huber (Paricá) in degraded sites under different regimes of preparation of area in the Microregion Guamá, Aurora do Pará-PA. Monograph of Specialization,
Federal University of Pará. Belém, Pará.. Monografia de especialização, Universidade Federal do Pará. Belém, Pará. (In Portuguese)

FAO. 1983. The recycling of organic matter in Agriculture. In Latin America. Report of the Latin American workshop meeting about recycling of organic materials in agriculture. Roma, Italia.. 215 $\mathrm{p}$ ( in Espanhol).

FAO. 2002. Carbon capture in the soils for better management of the land (reports about World Soil Resources). 60 pp (In Espanhol).

IBGE. Demographic cense. Available in www.ibge.gov.br. Acess in 03/28/2009. (In Portuguese).

Kern, D.C. 1996. Geochemistry and pedogeochemical of archaeological sites with Black Earth in the National Forest Caxiuanã (Portel-Pará). Belém-PA. Doctoral Thesis, Federal University of Pará, Belém, Pará, 124p. (In Portuguese).

Kern, D. C., Cometti, J.L. S., Ruivo, M. de L. P., Frazão, F.J. L., Rodrigues, T. E., Costa, M. L. da, Piccinin, J. L., Rocha, J. B. da.2006. New Dark Earth Experiment in the Tailândia City - Pará-Brazil: the Dream of Wim Sombroek In: 18th World Congress of Soil Science, 2006, Philadelphia. This presentation is part of 72: 1.6B Amazonian Dark Earth Soils (Terra Preta and Terra Preta Nova): A Tribute to Wim Sombroek - Oral. ( In English).

Loureiro, A.A.; Freitas J.A.de; Ramos ,K.B.; Freitas, L.C.A; 2000. Timber Essences of the Amazon, volume 4. Manaus. INPA. 114 p. (In Portuguese).

Monteiro, K.F.G. 2004. Use of Wood Waste as Cover in the Ground: The Case Study of an agroforestry system in the State of Pará. Masters dissertation, Universidade Federal Rural da Amazônia. Belém-Pará. 102 p. (In Portuguese whit in abstract in english).

Monteiro, K.F.G.; Kern, D.C.; Ruivo,M. L.P.; Rodrigues, T.E. 2009. Use of wood industry residues in soil as an alternative to improve the environmental conditions in an agroforestry system for reforestation, Tailândia, PA. p. 705-716. In. Porro, R. (Eds.). Agroforestry Alternative for the Amazon. Embrapa Technological Information - Brasilia-DF. (In Portuguese).

Monteiro, K.F.G.; Kern, D.C.; Rodrigues, T.E ;Ruivo,M. L.P.; Farias, P.R.S. 2006. Characterizing of the ywllow argisols whit addition of Woods residues: a use alternative as covering in soils of the amazon paraense. Boletim do Museu Paraense Emílio Goeldi. Ciências Naturais - Belém: MPEG- 1(1): 139-149. ( In portuguese whit abstract in english).

Ruivo, M. L. P.; Salazar, E. R. C. ; Monteiro, K. F. P.; Cordeiro, M. I. C. ; Piccinin, J. L. ; Oliveira, M. L. S. 2006. Evaluation of growth of paricá (Schizolobium parahyba var amazonicum (Huber ex Duke) Barneby in different agroforestry systems in northeastern of Pará. Congress of Agroforestry System, IV. SBSAF, Campus Gouytacazes. CD rom of the complete works. ( In portuguese whit abastract in english).

Recebido em 23/11/2007

Aceito em 02/12/2009 
